\title{
Application of cortical bone plate allografts combined with LISS plates for fixation of comminuted distal femur fractures
}

\section{Zhimin Guo}

the affiliated southeast hospital of xiamen university

\section{Conghui Shi}

the affiliated southeast hospital of Xiamen University of the West Indies at Saint Augustine

Hui Liu

the affiliated southeast hospital of xiamen university

\section{Zhida Chen}

the affiliated southeast hospital of xiamen university

Yongzhi Lin

the affiliated southeast hospital of xiamen university

Jin Wu ( $\nabla$ wuxinyu0102@163.com )

the affiliated southeast hospital of xiamen university

\section{Research article}

Keywords: Cortical bone plate allograft, Comminuted fracture Distal f emoral, Implant failure, LISS

Posted Date: February 17th, 2020

DOl: https://doi.org/10.21203/rs.2.23654/v1

License: (9) (i) This work is licensed under a Creative Commons Attribution 4.0 International License. Read Full License 

9

\section{Application of cortical bone plate allografts combined with LISS plates for fixation of comminuted distal femur fractures}

\section{Zhimin Guo ${ }^{1 \dagger}$, Conghui Shi ${ }^{2 \dagger}$, Hui Liu ${ }^{1}$, Zhida Chen ${ }^{1}$, Yongzhi Lin ${ }^{1}$, Jin Wu ${ }^{1 *}$}

1. Department of Orthopaedics, the Affiliated Southeast Hospital of Xiamen

University, Orthopaedic Center of People's Liberation Army, Zhangzhou, 363000, People's Republic of China.

2. Department of Emergency department, the Affiliated Southeast Hospital of Xiamen University, Zhangzhou, 363000, People's Republic of China

*Corresponding author: Jin Wu, Department of Orthopaedics, the Affiliated Southeast Hospital of Xiamen University, Orthopaedic Center of People's Liberation Army, Zhangzhou, 363000, People's Republic of China. Tel: 86-0596-2931538; Fax: 86-0596-2931538; Emails:wujin1983@xmu.edu.cn.

$\dagger$ Equal contributors. 4

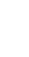

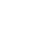
8

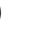




\section{Abstract}

2 Background: At present, the management of comminuted distal femur fractures

3 remains challenging for orthopedic surgeons. The aim of this study is to report a

4 surgical treatment for comminuted distal femur fractures using supplementary medial

5 cortical bone plate allografts in conjunction with the lateral less invasive stabilization

6 system (LISS) plates, and to discuss the therapeutic effects of this treatment after

7 long-term follow-up with patients.

8 Patients and methods: From January 2009 to January 2014, the records of thirty-three

9 patients who underwent supplementary medial cortical bone plate allografts combined with lateral LISS plate fixation were reviewed. Twenty-nine patients suffered from

11 closed fractures and four had open fractures. Clinical and radiographic data were collected during regular post-operative follow-up visits. Functional outcomes were determined according to the special surgery knee rating scale (HSS) used at the 14 hospital.

Results: Thirty patients were followed for 13 to 73 months after surgery, with an average follow-up time of 31.3 months. The mean time to bone union was 5.4 months

17 (range of 3-12 months) and the mean range of knee flexion was $105.6^{\circ}$ (range of $\left.80^{\circ}-130^{\circ}\right)$. Of the remaining patients, 10 had a score of "Excellent" while 10 had a 19 score of "Good" (the proportion of "Excellent" and "Good" scores was 67.7\%). Three patients had superficial or deep infections, one patient had nonunion that required

21 bone grafting and one patient had post-traumatic knee arthritis. No loosening of 22 fixation or refracture had occurred in any patient at the time of the last follow-up 
1 appointment.

2 Conclusions: Based on these promising results, we propose that supplementary medial

3 cortical bone plate allografts combined with lateral LISS plates fixation may be a

4 good treatment option for comminuted distal femur fractures. This treatment choice

5 not only resulted in markedly improved stability on the medial side of the femur, but

6 also satisfactory outcomes for distal femoral fractures.

7 Key words: Cortical bone plate allograft; Comminuted fracture; Distal femoral;

$8 \quad$ Implant failure; LISS

9

10

11

12

13

14

15

16

17

18

19

20

21

22 


\section{$1 \quad$ Introduction}

2 Distal femoral fractures comprise approximately 3\%-6\% of all femoral fractures [1].

3 Till now, effective treatment of comminuted distal femur fractures remains difficult

4 for orthopedic surgeons. These fractures are often unstable and comminuted, typically

5 resulting either from falls in female patients older than 75 years or as a result of the high energy activities common amongst adolescent boys and men aged 15 to 24 years

7 [2]. Classification of distal femur fractures was first described by Müller et al. and 8 expanded in the AO/OTA classification $[3,4]$. These classifications are based on 9 fracture location and pattern and are useful in determining treatment and prognosis.

With the development of improved internal fixation devices, operative treatment can now produce better results than nonoperative treatment. This is especially true for comminuted supracondylar and intercondylar femur fractures [5].

Surgical treatment of comminuted distal femur fractures demands experience and surgical skill. A complete set of instruments and familiarity with their use are required for this method of fixation. Condylar buttress plates, dynamic condylar screws, intramedullary nailing, LISS plates, and external fixation were introduced to facilitate the treatment of these types of fractures [6-9]. However, the spectrum of injuries is so great that no single implant has been found to be suitable for every case. Moreover, patient outcomes with these types of fractures are generally unsatisfactory due to the proximity of the fracture to the knee joint [10], meaning that regaining full knee motion and function may be difficult. In addition, internal fixation above such fractures is disadvantageous since a lengthy non-weight bearing period is required 
1 following surgery, and significant complications such as malunion, nonunion,

2 infection, and implant failure occur at relatively high rates in many reports [11-14].

3 Given these challenges, this study sought to investigate a novel surgical

4 treatment strategy for comminuted distal femur fractures. The fractures included in

5 this study were in accordance with AO/OTA classification, consisting of patients with

6 either type A3 fractures involving distal shaft comminution, type C2 fractures

7 involving metaphyseal comminution, or type C3 fractures characterized by

8 metaphyseal and intra-articular comminution. The approach described here features

9 the use of a supplementary medial cortical bone plate allograft in conjunction with a

10 lateral LISS plate. Therapeutic effects were assessed in patients, with an average 11 follow-up time of 31.3 months.

\section{Patients and Methods}

\section{Clinical data}

This study was a retrospective analysis of existing clinical cases and was approved by the institutional review board. Written informed consent was obtained preoperatively for all patients. Thirty-three patients (20 males and 13 females) were enrolled in the study between January 2009 and January 2014. The average age at enrollment was 44.5 years (range was 18-78 years). Follow-up visits were conducted with thirty patients between 13 and 73 months post-operation, with an average follow-up time of 31.3 months. One patient stopped responding after a 3-month follow-up visit, and two patients lost connection at 6-month follow-up visit. Twenty-nine patients suffered 
1 from closed fractures and 4 had open fractures (1 Gustilo I, 1 Gustilo II, 1 Gustilo

2 IIIA and 1 Gustilo IIIB). According to the AO/ASIF system, 33 fractures were

3 classified as the following: A3 $(n=10), C 2(n=13)$, and C3 $(n=10)$. The causes of

4 injury included: traffic accidents (20 patients, $60.6 \%$ ), heavy object crush injuries (5

5 patients, $15.1 \%$ ), falls from a significant height (6 patients, $18.2 \%$ ), and implant

6 failure (2 patients, 6.1\%). Two patients had fractures associated with an ipsilateral

7 tibial fracture (including one popliteal artery injury patient), two with a

8 hemopneumothorax, two with a traumatic brain injury, two with contralateral tibial

9 and fibula fractures, and one with an ipsilateral patella fracture. All patients were

10 diagnosed according to clinical presentation, X-ray, and computer tomography (CT)

11 scans. Study participants were evaluated post-operatively every 1-2 months in the

12 outpatient clinic. Detailed clinical patient parameters are shown in Table I.

\section{Pre-operative preparation}

Proximal tibial skeletal traction was performed immediately after all patients with closed fractures were admitted to the hospital. Patients with Gustilo I and Gustilo II open fractures first underwent debridement and suturing, after which they received proximal tibial skeletal traction. In two patients with a Gustilo III fracture, limited internal fixation combined with external fixation was implemented following debridement. All patients with open fractures received postoperative intravenous antibiotics for 24 to $48 \mathrm{~h}$. X-ray and CT examinations were used to visualize fracture displacement and the presence of fragments when determining the surgical strategy 
1 for each patient. All patients underwent surgical treatment as soon as their condition

2 had stabilized.

3

\section{Surgical procedure}

5 Prophylactic antibiotics were given $30 \mathrm{~min}$ prior to surgery. No tourniquet was used.

6 The patient was placed under either general or spinal anesthesia and then positioned in

7 a supine position with a bolster under the knee to acquire $20^{\circ}-30^{\circ}$ of flexion. This was

8 done in order to relax the deforming force of the gastrocnemius. For type A3 fractures,

9 a 4-5 cm lateral incision was made just proximal to the joint line. A distal femoral

LISS plate (AO, Synthes Inc. West Chester, Pennsylvania, USA) was slipped under the vastus lateralis proximally and provisionally fixed distally using $\mathrm{K}$ wires. Close reduction was accomplished using traction and external manipulation and confirmed under fluoroscopy. During the procedure, specific attention was paid to limb alignment and length. When the position of the LISS plate was deemed satisfactory, three to six locking screws were inserted in the distal and proximal part of the bone respectively.

For type C2 and C3 fractures, an incision made on the lateral condyle of the femur and elongated to the tibial tubercle to fully expose the anterior and lateral aspects of the femoral condyle. Intercondylar fractures were then reduced and fixed with cannulated screws (AO) to form the supracondylar fracture. These fractures were then treated as type A3 fractures. For patients with implant failure after surgery, the lateral parapatellar approach was used to remove the implant. After the fracture was 
1 fully exposed, any scar tissue and sclerotic bone was excised and the medullary cavity

2 was reamed. An appropriate length LISS plate was then used to fix the fracture, and

3 autologous iliac bone was implanted.

A suitable width cortical bone plate allograft (Xin Kang Chen Medical

5 Technology Development Co., Ltd, Beijing, China) was selected and trimmed with a

6 wire saw. The sharp edge of the cortical bone plate allograft was filed with a bone file,

7 and the tip was rounded and obtuse. A 4-5 cm anteromedial incision was made along

8 the anterior margin of the pes anserinus, following the adductor canal. The fascial

9 envelope surrounding the vastus medialis along the posterior margin of the muscle

10 was then incised. Blunt dissection was used to elevate the muscle off the periosteum

11 and the intermuscular septum from the adductor tubercle to the intact proximal

12 femoral shaft. Next, a periosteal elevator was used to strip the region between the

13 periosteum and adductor muscles of the thigh. The prepared cortical bone plate

14 allograft was implanted via the anteromedial incision and placed on the opposite side

15 of the LISS plate. The LISS plate and cortical bone plate allograft were fixed in place

16 with cortical bone screws. At least two screws were used at the distal and proximal

17 ends of the bone plate. Finally, the open wound was rinsed and the incisions were

18 closed, with a suction drain at the surgical site.

\section{Postoperative management}

21 All patients received post-operative intravenous antibiotics for $24 \mathrm{~h}$. Suction drains were removed on day 2-3. Active and passive range-of-motion exercises were then 
started. Full weight-bearing activity was allowed after a bridging callus was observed on radiographs.

\section{Outcome assessment}

Outcomes after surgery were evaluated according to HSS scores, which rely on a 100-point scoring system that assesses pain, function, range of motion, muscle strength, flexion deformity, and joint stability. Overall, "Excellent" was classified as a cumulative score of 85 or more, "Good" as 70 to 84, "Fair" as 60 to 69 and "Poor" as

60 or less. Postoperative functional results were obtained regularly. Postoperative radiological parameters, including X-rays and CT scans, were taken every four weeks to evaluate bony fusion.

\section{Results}

The mean non-weight-bearing period was 10.7 weeks (range was 8-15 weeks) and mean time to bone union (formation of a circumferential bridging callus across the fracture) was 5.4 months (range was 3-12 months). Three patients stopped visits and ceased communication during the follow-up period (Patients 21, 28, and 33). Outcomes for the remaining patients were "Excellent" for 10 and "Good" for 10 , making the percentage of combined "Excellent" and "Good" scores 67.7\%. The mean range of knee flexion was $105.6^{\circ}$ (range of $80^{\circ}-130^{\circ}$ ). More specifically, 2 patients had an $80^{\circ}$ range, 4 patients had a $90^{\circ}$ range, 7 had a $100^{\circ}$ range, 12 had a $110^{\circ}$ range, 
1 and 5 had $\geqslant 120^{\circ}$ range of knee flexion. All patients achieved full knee extension.

2 Three patients had weakness in their quadriceps, but all others attained full quadriceps

3 strength. Six patients had the implant removed (Table II).

One patient had a deep infection five days after the operation and underwent a secondary surgery (implant removal and external fixation). There were two patients who had minor surgical complications, including one superficial wound infection and one partial wound dehiscence. After debridement and suturing, both patients' complications were resolved. One patient with nonunion required bone grafting without hardware exchange. Post-traumatic arthritis was seen in one patient at the final follow-up, which was based on the radiologic assessment (Table II). Typical cases are shown in Fig. 1 (Patient 2), Fig. 2 (Patient 5), and Fig. 3 (Patient 10).

\section{Discussion}

Comminuted distal femur fractures are frequently associated with severe comminution, substantial soft tissue injury, and bone defects. Prior to the 1970s, non-operative management was the treatment of choice [2]. With the steady improvement of surgical techniques and implants, operative fixation has gained widespread acceptance. Historically, these fractures were treated with condylar buttress plates [6]. Gradually, retrograde nails and dynamic condylar screws took the place of condylar buttress plates. This shift was due to their superior biomechanical design that resulted in decreased varus collapse events when compared with the results using standard condylar buttress plates [15]. Recently, locking plates have become the main treatment for comminuted distal femur fractures, particularly for supracondylar and 
1 intercondylar comminuted femur fractures. With the increased number of fixation

2 screws used in the distal femur metaphysis, locking plates provide increased

3 biomechanical resistance and stability [16]. However, perioperative and postoperative

4 complications such as malunion, nonunion, implant failure, and infection are still

5 common with this approach [14].

The main reasons for implant failure are primarily due to the following problems: (1) high bending stress exerted on the laterally placed plates in the presence of marked cortical defects and (2) locking plates are usually implanted using the minimally invasive percutaneous plate osteosynthesis (MIPPO) technique. Since the MIPPO technique is relatively short range and intraoperative fluoroscopy has a limited range, there is a high incidence (approximately 30\%) of axial malalignment after surgery. Axial malalignment results in increased load on the plate, which can cause implant failure. Here, implant failure was found in two patients over the age of 60 who had been initially treated with a single-side plate and screws, followed by additional operations as needed. The current treatment approach for implant failure features scar tissue removal and large amounts of autologous iliac bone grafts, as well as implant replacement. Bilateral autologous iliac bone grafts have often been applied to repair cortical defects, which can increase surgical trauma and the chance of infection. Furthermore, the stability immediately following the structural allograft cannot support early postoperative functional exercise, which is important for recovery. Therefore, we performed a medial implant of the cortical bone plate allograft integrated with a lateral LISS plate for the two patients with implant failures. Patient 2 was a 61-year-old male patient with a type A3 fracture that had been initially treated using dynamic condylar screws. The implant failure was observed seven months following surgery and required reoperation. After treatment with a cortical bone plate 
1 allograft combined with LISS plate fixation, bone union was observed five months 2 later (Fig. 1).

Types C2, C3, and partial A3 fractures of the distal femoral are prone to induce nonunion and implant failure, particularly in the cases of severe cortical defects in the medial femur. On the basis of lateral LISS plate implantation using MIPPO technology, a suitable length and width allogeneic cortical bone plate was implanted from the medial epicondyle of the femur, which achieved an integrated fixation of the triangular support and avoided excessive elevation of the periosteum at the fracture site. For severe comminuted fractures and/or periprosthetic fractures of the distal femur, double plating with autogenous bone grafting executed via a modified Olerud extensile approach was also used. Although acceptable clinical outcomes were achieved, there are some limitations to this approach, including excessive elevation of the periosteum, large trauma (tibial tuberosity osteotomy), and lack of integrated fixation [17]. Recently, a double-plating technique was used for the treatment of supracondylar femur fractures. Based on promising follow-up results, they recommended this technique specifically for patients with poor bone quality, comminuted fractures, and very low periprosthetic fractures [18]. However, no detailed functional outcomes were described in their results and some important points were needed to be clarificated [19] .

The application of allogeneic cortical bone plates in repairing bone defects has been frequently reported and satisfactory clinical results have been achieved with this approach [20,21]. In our study, an allogeneic cortical bone plate was used in the treatment of comminuted distal femur fractures and has the following advantages: (1) wide scope of application; (2) the union of the allogeneic cortical bone plate and host bone can reconstruct cortical defects of the medial femur, and when combined with an 
1 autologous iliac bone graft, this treatment has a strong osteoinductive effect and can

2 promote bone healing; (3) LISS plates and allogeneic cortical bone plates were

3 implanted using MIPPO technology, which minimized periosteal elevation and

4 disruption of blood supply at the fracture site, which not only increased fixation

5 rigidity, but also contributed to fracture healing; (4) allogeneic cortical bone plates are

6 a biomechanically sound alternative to metal plates fixed with screws, and could

7 markedly improve stability and rigidity after lateral LISS plate fixation; (5) utilization

8 of a LISS plate and allogeneic cortical bone plate presented firm integrated fixation of

9 the triangular support. Furthermore, knee function exercises were conducted soon

10 after the operation, resulting in overall better therapeutic outcomes.

\section{Conclusion}

12 We recommend that a cortical bone plate allograft combined with the LISS plate

13 fixation technique be used for treatment of comminuted distal femur fractures. This is 14 especially indicated in cases of severe medial femur cortical defects and implant 15 failure after surgery. However, there are some limitations to this study, including its retrospective nature as well as the relatively small group of patients studied. A long-term study with a larger number of patients and control groups that include other fixation methods should be performed to further validate our findings here.

\section{List of abbreviations}

CT, computer tomography; HSS, special surgery knee rating scale; LISS, less invasive stabilization system; MIPPO, minimally invasive percutaneous plate osteosynthesis. 


\section{Acknowledgements}

2 We thank all participants in this study for their enthusiasm, tireless work, and

3 sustained support.

4

$5 \quad$ Funding

6 The author(s) disclosed receipt of the following financial support for the research,

7 authorship, and/or publication of this article: This work was supported by grants from

8 the General Program of PLA Nanjing Military Area Command (MS096).

9

Availability of data and materials

11 confidentiality.

Ethics approval and consent to participate

This study was a retrospective and clinical study and approved by the Xiamen University institutional review board. Written informed consent was obtained from all patients preoperatively.

\section{Consent for publication}

All authors are in agreement with the content of the manuscript and have approved the manuscript for submission. 
1 The author(s) declared no potential conflicts of interest with respect to the research,

2 authorship, and/or publication of this article.

3

4 Authors' contributions

$5 \quad$ ZG: Writing the paper, data analysis and collection.

6 CS: Searching the related articles, data analysis.

$7 \quad$ HL: Data collection.

$8 \quad$ ZC: Writing the paper, data analysis and collection.

9 YL: Searching the related articles, data analysis.

10 JW: Data collection, performed surgeries, research team manager.

11 All authors have contributed significantly and are in agreement with the content of the 12 manuscript. 
1 Fig. 1. Representative images of patient 2 (61-year-old male patient with a type A3

2 fracture that had been initially treated with dynamic condylar screws). Implant

3 breakage was observed seven months after surgery $(a, b)$. X-ray at 5 months after

4 operation. Bone union was observed (c, d). Follow-up X-ray at 36 months (e, f).

$5 \quad$ Follow-up X-ray at 60 months (g, h). Follow-up X-ray at 73 months (i, j).

7 Fig. 2. Representative images of patient 5 (29-year-old male who suffered a heavy 8 object crush to his left thigh). X-ray at admission (a, b). X-ray 5 days after the 9 operation (c, d). Follow-up X-ray at 3 months (e, f). Follow-up X-ray at 30 months (g, h). Full-length radiography showing the lower limb at a 33 month follow-up visit. Limb alignment and length was good (i). Range of knee joint motion at a 33 month follow-up visit. The patient achieved full knee extension. However, the range of knee flexion was only $90^{\circ}(\mathrm{j}, \mathrm{k})$.

Follow-up X-ray at 69 months $(\mathrm{k}, 1)$.

Fig. 3. Representative images of patient 10 (21 year old male patient with a Gustilo IIIB fracture resulting from a traffic accident). X-ray at admission (a, b). X-ray after emergency operation. Limited internal fixation combined with external fixation was utilized (c, d). X-ray 3 months after an interfixation operation (e, f). Follow-up X-ray at 9 months (g, h). Follow-up X-ray at 12 months. Removal of the tibial implant (i, j). 


\section{1}

\section{References}

1. Court-Brown CM, Caesar B. Epidemiology of adult fractures: a review. Injury. 2006;37(8):691-7.

2. Martinet O, Cordey J, Harder Y, Maier A, Bühler M, Barraud GE. The epidemiology of fractures of the distal femur. Injury. 2000;Suppl3:62-3.

3. Müller ME, Nazarian S, Koch P, Schatzker J. The Comprehensive Classification of Fractures of Long Bones. Berlin, Heidelberg, New-York: Springer-Verlag.1990.

4. Marsh JL, Slongo TF, Agel J, Broderick JS, Creevey W, DeCoster TA, et al. Fracture and dislocation classification compendium-2007: Orthopaedic Trauma Association classification, database and outcomes committee. J Orthop Trauma. 2007;21(10 Suppl):S1-S133.

5. Johnson KD. Internal fixation of distal femoral fractures. Instr Course Lect. $1987 ; 36: 437-48$.

6. Davison BL. Varus collapse of comminuted distal femur fractures after: open reduction and internal fixation with a lateral condylar buttress plate. Am J Orthop (Belle Mead NJ). 2003;32(1):27-30.

7. Narsaria N, Singh AK, Rastogi A, Singh V. Biomechanical analysis of distal femoral fracture fixation: dynamic condylar screw versus locked compression plate. J Orthop Sci. 2014;19(5):770-5.

8. Kulkarni SG, Varshneya A, Kulkarni GS, Kulkarni MG, Kulkarni VS, Kulkarni RM. Antegrade interlocking nailing for distal femoral fractures. J Orthop Surg 
2 9. Smith TO, Hedges C, MacNair R, Schankat K, Wimhurst JA. The clinical and

3 radiological outcomes of the LISS plate for distal femoral fractures: a systematic

4 review. Injury. 2009;40(10):1049-63.

5 10. Ali F, Saleh M. Treatment of isolated complex distal femoral fractures by external fixation. Injury. 2000;31(3):139-46.

11. Wang MT, An VVG, Sivakumar BS. Non-union in lateral locked plating for distal femoral fractures: A systematic review. Injury. 2019;50(11):1790-1794.

12. Rollo G, Pichierri P, Grubor P, Marsilio A, Bisaccia M, Grubor M, et al. The challenge of nonunion and malunion in distal femur surgical revision. Med Glas (Zenica). 2019;16(2). [Epub ahead of print].

13. von Keudell A, Shoji K, Nasr M, Lucas R, Dolan R, Weaver MJ. Treatment Options for Distal Femur Fractures. J Orthop Trauma. 2016;30 Suppl 2:S25-7.

14. Henderson CE, Kuhl LL, Fitzpatrick DC, Marsh JL. Locking plates for distal

15. Hartin NL, Harris I, Hazratwala K. Retrograde nailing versus fixed-angle blade plating for supracondylar femoral fractures: a randomized controlled trial. ANZ J Surg. 2006;76(5):290-4.

16. Frigg R, Appenzeller A, Christense R, Frenkl A, Gilbertz S, Schavanl R. The development of the distal femur less invasive stabilization system (LISS). Injury. 2001;32 Supp 13:SC24-31. 
1 17. Khalil Ael-S, Ayoub MA. Highly unstable complex C3-type distal femur

2 fracture: can double plating via a modified Olerud extensile approach be a 3 standby solution?. J Orthopaed Traumatol. 2012;13(4):179-88.

4 18. Steinberg EL, Elis J, Steinberg Y, Salai M, Ben-Tov T. A double-plating approach 5 to distal femur fracture: A clinical study. Injury. 2017;48(10):2260-5.

6 19. Kumar P, Patel S, Kumar V, Rajnish RK, Hooda A. A double-plating approach to 7 distal femur fracture: A clinical study; how apt is the technique? How strong is $8 \quad$ the evidence? Injury. 2018;49(3):737-38.

9 20. Shih HN, Shih LY, Cheng CY, Hsu KY, Chang CH. Reconstructing humerus grafting. J Orthop Trauma. 2005;19(1):36-42. 
Figures
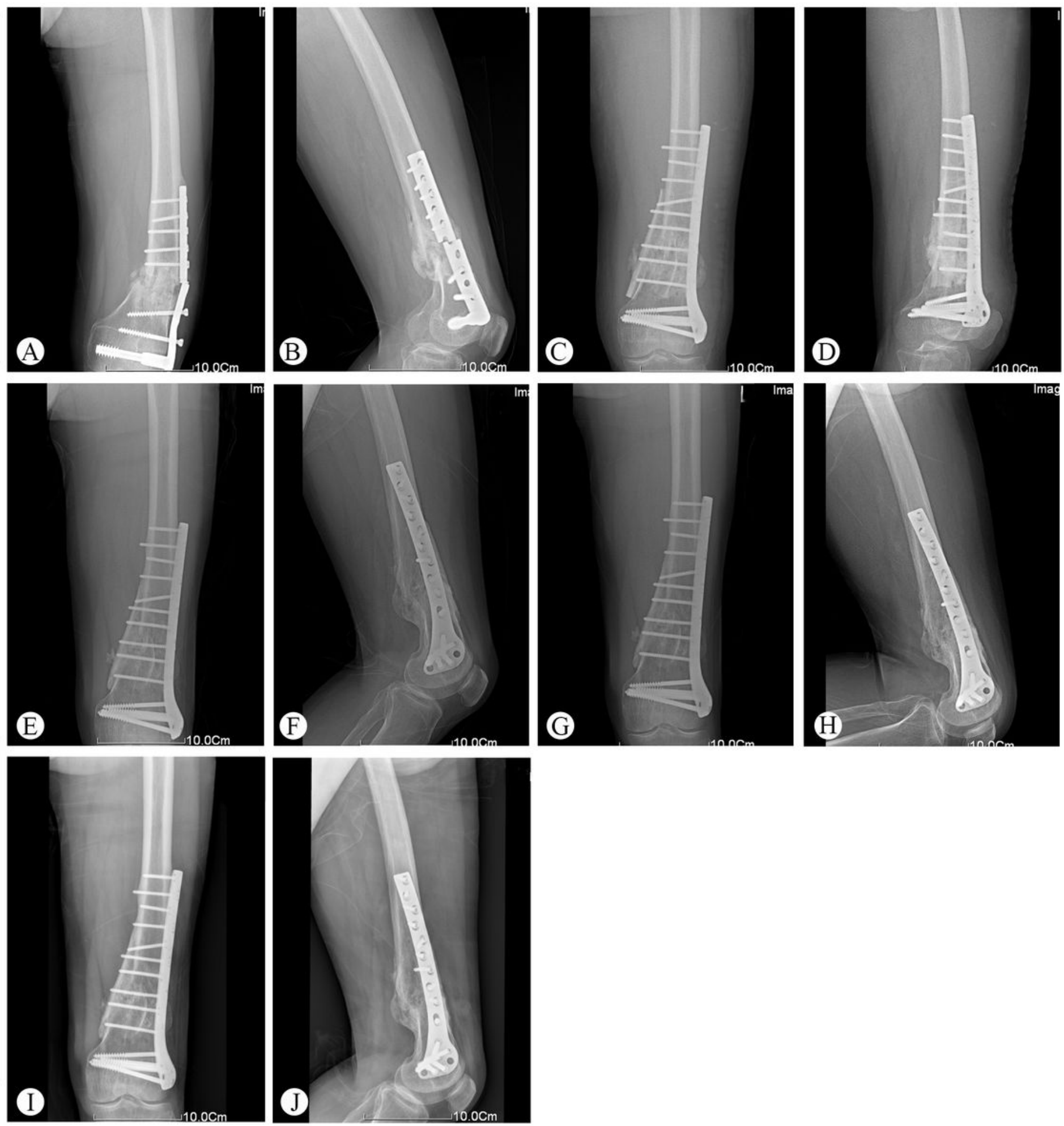

Figure 2

Representative images of patient 2 (61 year ol d male patient with a t ype A3 fracture that had been initially treated with dynamic condylar screws screws). Implant breakage was observed seven months 
after surgery $(\mathrm{a} b)$. X-ray at 5 months after operation. Bone union was observed c, d Follow-up X-ray at 36 months $(e, f)$. Follow-up X-ray at 60 months $(g, h)$. Follow up X-ray at 73 months $(i, j)$.
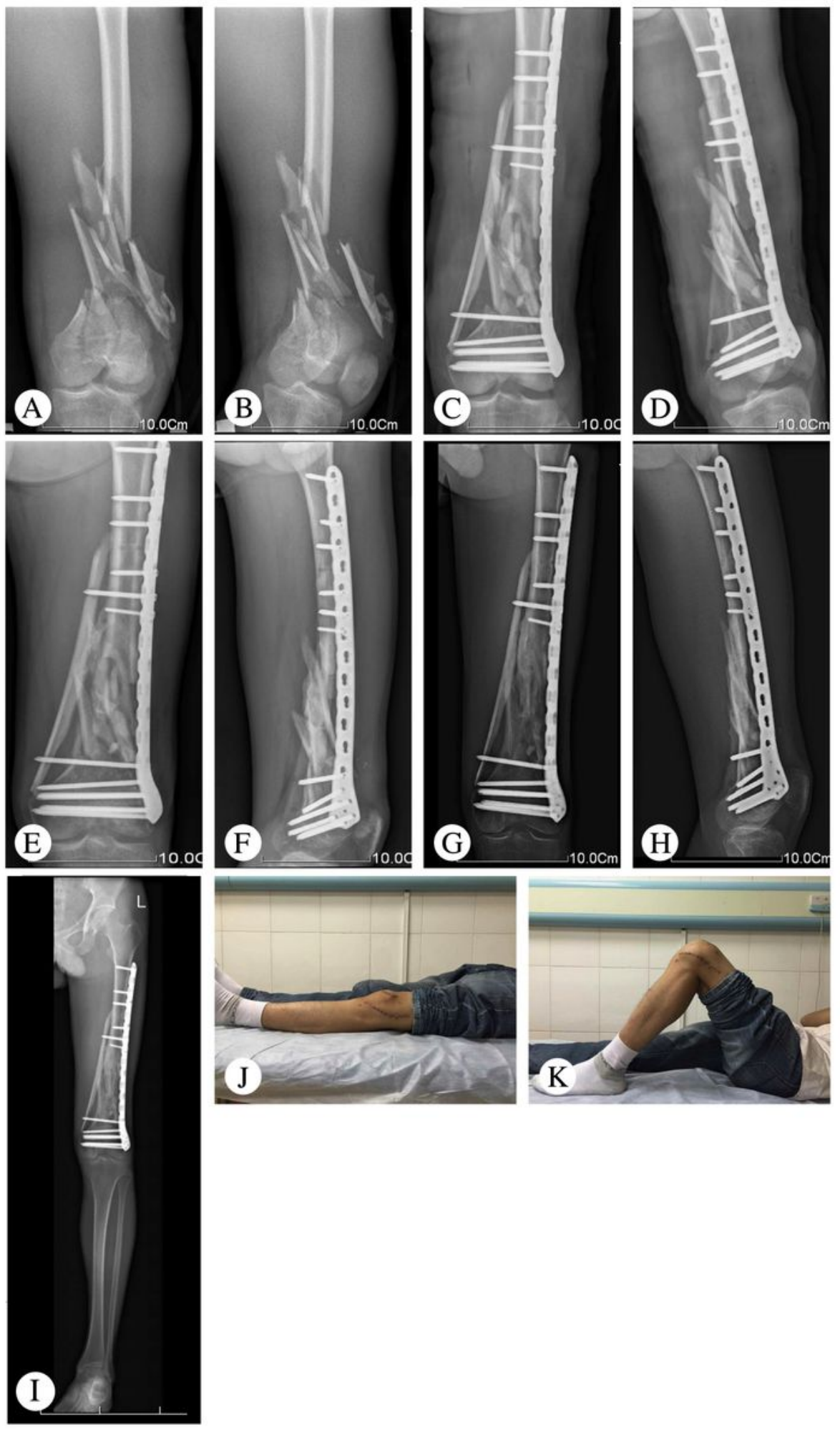

\section{Figure 3}

Representative images of patient 5 (29-year-old male who suffered a heavy object crush to his left thigh). X-ray at admission ( $a$ b ). X-ray 5 days after the operation (c, d). Follow-up X-ray at 3 months (e, f). Followup X-ray at 30 months $(g, h)$. F ull length radiography showing the lower limb at a 33 month follow-up 
visit. Limb alignment and length was good (i). Range of knee joint motion at a 33 month follow-up visit. The patient achieved full knee extension. However, the range of knee flexion was only $90^{\circ} \mathrm{j}, \mathrm{k}$
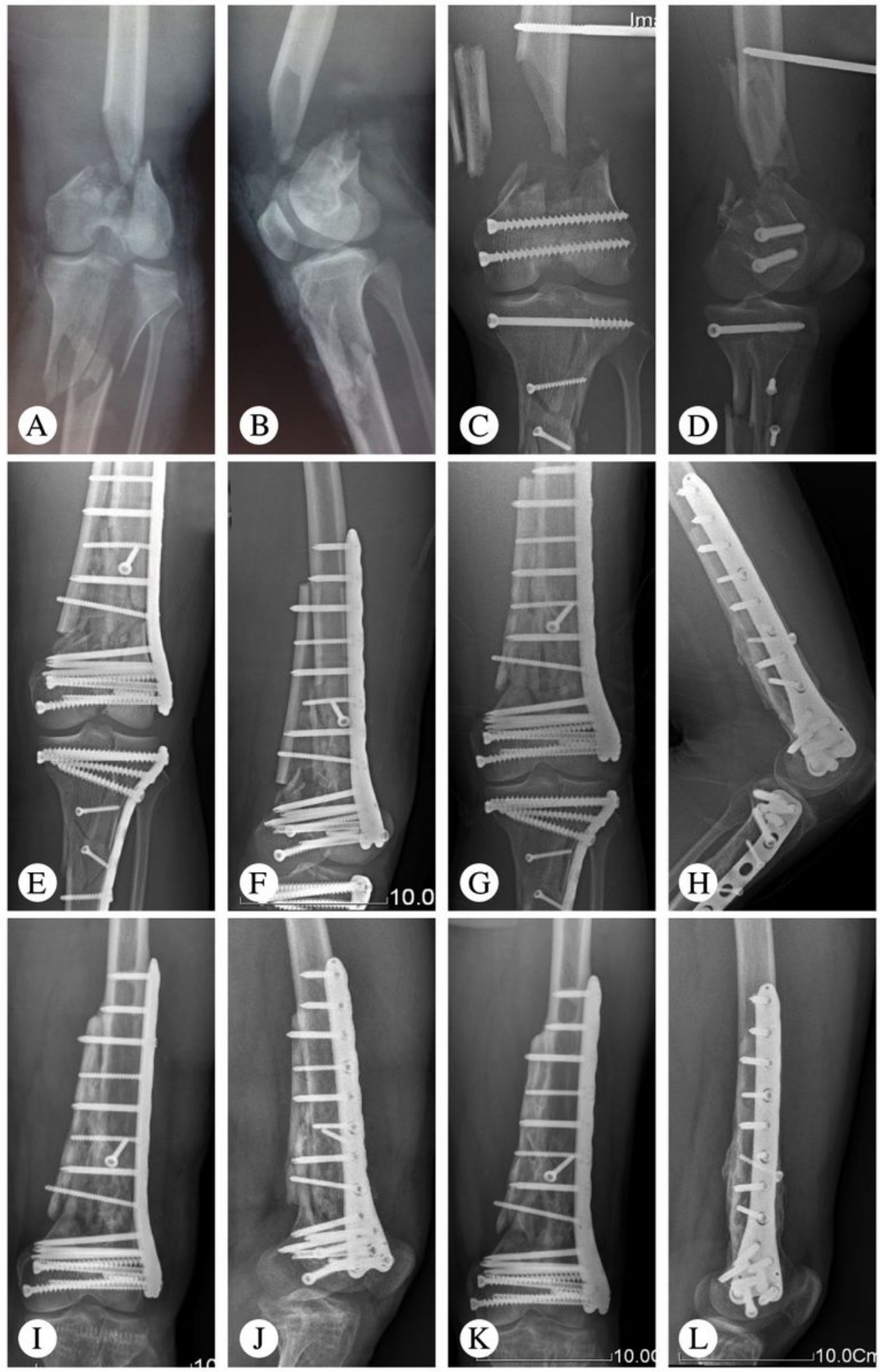

Figure 6

Representative images of patient 10 (21 year old male patient with a Gustilo IIIB fracture resulting from a traffic accident). X-ray at admission ( $a, b)$. X-ray after emergency operation. $L$ imited internal fixation combined with external fixation was utilized (c d) X-ray 3 months after an interfixation operation (e f). 
Follow-up X-ray at 9 months ( $\mathrm{g} \mathrm{h}$ ). Follow up X-ray at 12 months. Removal of the tibial implant (i,j). Follow-up X-ray at 69 months $(k, l)$

\section{Supplementary Files}

This is a list of supplementary files associated with this preprint. Click to download.

- Tablell.pdf

- Tablel.pdf

- Tablell.pdf

- Tablel.pdf 